\title{
UNA DISCUSIÓN ACTUAL ¿GRAVE O SEVERO?
}

\section{GRAVE OR SEVERE? A CURRENT DISCUSSION}

\author{
MURUBE $\mathrm{J}^{1}$, MUÑOZ-NEGRETE $\mathrm{F}^{2}$, ARRUGA J ${ }^{3}$
}

La actual generación adulta siempre denominó «grave» al grado más lesivo de un cuadro clínico. Paulatinamente los médicos jóvenes han empezado a substituirlo por «severo», provocando generalmente una actitud de rechazo en la generación médica adulta. Así ha surgido una pequeña confrontación lingüística generacional, en la que el aparente ganador es el mayor, porque suele ocupar un puesto directivo y de respeto en la enseñanza y en los servicios médicos. Pero el real ganador será el joven, porque ineludiblemente, arrastrado por el viento de estos tiempos, la terminología médica y después la general irán incorporando más y más el adjetivo severo.

\section{¿QUÉ SIGNIFICARON GRAVIS Y SEVERUS EN LATÍN?}

En primer lugar digamos que en el lenguaje no existen sinónimos absolutos, pues dos términos considerados sinónimos siempre tienen connotaciones distintas: papá no es sinónimo absoluto de padre, pues el primero tiene una connotación de niñez y cariño que falta al segundo. Ni oculista es sinónimo absoluto de oftalmólogo; ni anestesista de anestesiólogo, pues lo primero tiene una connotación de antigüedad y lo segundo de modernidad y de mayor eficacia.

En segundo lugar, un término tiene generalmente varias acepciones, y puede ser sinónimo de otro en alguna de ellas, pero no en las otras. Coser es sinónimo de suturar cuando se refiere a coser o suturar una herida, pero no cuando se refiere a coser un botón o un traje. Estas variaciones del significado se hacen mayores cuando se trata de adjetivos, pues aplicados a un substantivo pueden significar una cosa y aplicados a otro, pueden significar incluso lo contrario que en el primer caso

En tercer lugar, el significado de un término evoluciona con el tiempo y los hábitos, incluso dentro de la vida de una generación. Bárbaro se usó en la Grecia clásica para los pueblos extranjeros; más tarde pasó en Roma para los pueblos que la circundaban y acabaron terminando con su imperio romano; después añadió la acepción de salvaje y cruel; y hace unas décadas pasó también a significar excelente y magnífico.

Los romanos usaron los términos gravis y severus con significados varios, que a veces fueron coincidentes para determinadas acepciones, a veces parecidas aunque con matices algo distintos, y a veces claramente diferentes. A lo largo de los 12 siglos de existencia de la monarquía, república e imperio romanos ambos términos evolucionaron, modificaron algo sus significados, y se les añadieron acepciones nuevas.

El adjetivo gravis significó inicialmente «con peso», y a lo largo de la evolución del latín, tomó diversos significados según el objeto, persona o situación al que adjetivase, cambiando también esto con el siglo, la geografía y la circunstancia. En el diccionario Español-Latino de Blánquez grave se traduce como gravis, molestus, durus, a más de otras variantes. Así recogemos: gravitas armorum (Cæs), el peso de las armas; gravida mulier (Ter., Cic.), mujer encinta, gravis annis (Hor.), cargado de años; gravis sopor (Lucr.), sueño profundo, gravis morbus (Plan., Nep.), enfermedad mortal; gravis civitas (Cic.), ciudad importante; grave vulnus (Cæs.), herida grave; gravis sententia (Cic.), sentencia grave; gravia vectigalia (Suet.), impuestos onerosos; gravis culpa (Ov.), falta grave; grave edictum (Liv.), edicto severo; graviter olore (Mart.),

\footnotetext{
1 Catedrático Emeritus de Oftalmología. Universidad de Alcalá de Henares. Madrid. España.

E-mail: murubejuan@terra.es

2 Profesor Titular de Oftalmología. Jefe del Servicio de Oftalmología del Hospital Ramón y Cajal. Madrid. España.

3 Profesor Titular de Oftalmología. Universidad de Barcelona. Campus de Bellvitge. Barcelona. España.
} 
olor fuerte; gravior halitus (Plin.), mal haliento. El adjetivo pasó también a la fonología y la gramática, de forma que syllaba gravis (Quint.) es la sílaba sin acento agudo. E incluso adquirió significados con sentidos fuertemente positivos... graviter amare (Plaut.), amar mucho $(1,2)$.

El adjetivo severus también tuvo en latín múltiples significados. Sus acepciones son muchas y diversas, según la situación: severus in filium (Cic.), cruel con el hijo; pelagi severa (Lucr.), mares peligrosos; severa frons (Plaut.), frente ceñuda; severissimos judices (Cic.), jueves severísimos; severi custodes (Cic.), guardianes severos. A veces figuran como sinónimos del término serius, que a su vez tiene infinidad de matices en su significado: seria mala (Horac.), graves males; serius sermo (Suet.), lenguaje severo... Como vemos, severus tuvo también significados de grave, cruel, acerbo, serio, difícil, compatibles con los grados avanzados de un padecimiento o enfermedad

\section{¿CÓMO EVOLUCIONÓ EN ROMANCE ESPAÑOL?}

El diccionario de Alfonso de Palencia (1490) (3) da varias decenas de significados de grave según el substantivo al que se aplique: fuerte, serio, molesto, lleno, lleno de fruto, autoritario, necesario, doloroso, molesto, dañoso... pero no pone ningún ejemplo aplicada a las enfermedades.. En cuanto a severo pone como significados: triste, muy viejo y grave. Tampoco pone ningún ejemplo aplicado a las enfermedades.

El vocabulario español latino de Elio Antonio de Lebrija (1495) (4) hace semejantes a grave y severo, y así traduce grave cosa por gravis y por molestus, y gravedad por gravitas y molestia. Y severo por gravis y por molestus, severidad por gravitas, y severamente por graviter y por severiter.

El Diccionario de Autoridades (1734) (5) da nueve acepciones distintas al término grave: pesado, grande, circunspecto-severo, autoritario, altivo, serio, importante, molesto y sonido musical bajo. Y añade tras la última acepción: Ponerse grave: severitatem affectare (afectar severidad). Para severo (1939) da tres acepciones: riguroso-áspero-duro, exacto-puntual-rígido, y grave-serio-mesurado.

El diccionario de la Real Academia Española de 1780 (6) pone de grave diez acepciones: pesado, grande, circunspecto, autoritario, altivo, serio, importante, molesto, de sonido bajo, afectado... pero no pone entre sus ejemplos ninguno referido a la enfermedad. De severo pone tres acepciones: riguroso-áspero-duro, exacto-puntual-rígido en las leyes, y grave-serio-mesurado.

El Diccionario Castellano de Terreros y Pando (1788) (7) da del adjetivo grave diez acepciones: aplomado, pesado (física), de acento opuesto al agudo (gramática), bajo (música), majestuoso (moral), sabio y juicioso, molesto y peligroso (como grave enfermedad), importante, grave (delito), y soberbio. Y del adjetivo severo da dos acepciones: áspero-duro-intratable-riguroso, y exactoreligioso-observador de las leyes.

El penúltimo diccionario de la lengua española de la RAE (1992) (8) pone 14 acepciones de grave, de las que una es enfermedad grave, para la que es de cuidado. Y más adelante dice: lesión grave: la que incapacita para trabajar más de 30 días. Y para severo da tres acepciones: riguroso-áspero-duro, exacto-rígido y grave-serio-mesurado. Y de serio da seis acepciones: grave y compuesto en el modo de proceder, severo en el semblante, sincero y sin engaño, grave (como enfermedad seria), contrapuesto a jocoso, y sin engaño o burla. El último diccionario de la RAE (2001) (9) pone de grave diversas acepciones: que pesa, enfermo de cuidado, estilo circunspecto, serio, molesto, enfadado, sonido opuesto al agudo. Y de severo pone tres significados: riguroso-áspero-duro en el trato, exactorígido en la observación de las leyes, y cuando se aplica a una estación del año, que tiene temperaturas extremas.

Hemos revisado algunas obras de medicina en español de los siglos XIX y XX, escritas por autores españoles o iberoamericanos (10-15) y vemos que el término que usan para expresar el grado avanzado de una enfermedad es «grave».

\section{¿CÓMO EVOLUCIONÓ EN INGLÉS?}

A mediados del siglo $\mathrm{V}$ dC, tras la caída del imperio Romano, pueblos germánicos de tribus anglas, sajonas y jutas procedentes de Escandinavia y Jutlandia, invadieron el sudeste de la isla de Britannia, desalojando a los celtas que allí vivían. Poco a poco formaron una monarquía cuya lengua era de raíz germánica. En 1066 los franconormandos, de lengua francesa, les vencieron en la batalla de Hastings e instauraron un reino. El lenguaje de la aris- 
tocracia y de la gente más cultivada era el francés de la época, proveniente del latín, y poco a poco el pueblo vencido lo fue absorbiendo, de forma que la lengua inglesa de hoy día es básicamente una mezcla de raíces latinas y germánicas. Entre estos términos de raíz latina entraron los de gravis y severus. Gravis evolucionó a grave, con significados de weighty, serious, important... Y severus pasó a severe, con significados de austere, harsh, rigorous, violent, extreme... (17).

A lo largo de la historia, para definir la agresividad de un padecimiento médico se fue usando más y más el término severe, aunque no quedaron excluidos los otros. El inglés, una lengua que en tiempos de Shakespeare (1564-1616) sólo hablaban 6 millones de personas confinadas en el sur de Gran Bretaña (18), fue extendiéndose al norte de América en los siglos siguientes. El crecimiento de sus 13 colonias norteamericanas dio origen a los EEUU de América, que independizados a finales del siglo XVIII, fueron, casi dos siglos más tarde, los principales ganadores de la segunda guerra mundial (1939-1945). Con ello, la lengua inglesa dio un vuelco grande y pasó de ser la cuarta lengua de influencia en Europa en el momento de máxima expansión Victoriana (1837-1901), a hacerse la primera lengua mundial de difusión de la cultura y la interrelación. Poco a poco los congresos internacionales, que generalmente eran trilingües o multilingües, pasaron hacia los años 1960 y 1970 a hacerse monolingües en inglés/americano, y la literatura científica se fue haciendo cada vez más en inglés. Hoy día es un hecho consumado.

Los múltiples significados del adjetivo severe, cuando se aplica a las enfermedades, se refieren a su gravedad, y así pasa a expresarse cada vez más frecuentemente en las lenguas romances, consolidando un significado que había ido perdiendo importancia. Esta re-entrada de términos latinos procedentes del inglés americano es actualmente muy frecuente. Por ejemplo, del latín superficies, formada de las raíces super y facies para designar la parte o cara superior o exterior de un objeto, se formó las palabra francesa surface, y de ella, manteniendo la grafía pero cambiando la pronunciación, la inglesa surface. Hoy se reintroduce en las lenguas derivadas del latín en términos como surfactante (disminuidor de la tensión superficial), surfodacriología (ciencia de la lágrima en relación con la superficie ocular) y surfing (deporte de deslizamiento sobre el agua).

\section{LA DISPARIDAD}

El diccionario etimológico de Corominas (1983) (19) indica que la aplicación creciente del término severo a la gravedad de una enfermedad es un «grosero anglicismo», y añade que proviene de la influencia de la prensa y literatura americanas. También, el diccionario común de la Real Academia Española y de la Asociación de Academias de la Lengua Española (2005) (20) dice de severo que «...debe evitarse su uso como sinónimo de grave, importante o serio, calcos rechazables del inglés severe».

Sin embargo, el Diccionario del Español Actual, de Seco et al. (2005), (21) pone de severo cuatro significados: exigente, duro/riguroso, serio/austero y grave/importante. Y añade en esta cuarta acepción que se usa especialmente en medicina, y pone como ejemplo «alteraciones electromiográficas severas».

Como vemos, este intrascendente litigio sigue en pie.

\section{NUESTRA OPINIÓN}

Creemos que el que los jóvenes, acostumbrados a leer en inglés, ya sea en literatura anglo-americana o de otras procedencias, digan cada vez más frecuentemente que una enfermedad es severa, no debe ser rechazado:

1) Porque recupera uno de los significados que antaño tuvo, y aunque lleva en nuestra lengua varios siglos substituido por grave cuando se refiere a padecimientos médicos, vuelve a recuperarse por influencia angloamericana.

2) Porque la lengua evoluciona, y en sus nuevas incorporaciones va enriqueciéndose al matizar los cambios provocados por las incorporaciones. Por ejemplo: cuadra, recinto para animales y carros, pasó en una de sus acepciones a cochera, del magyar kocsi, coche, y de aquí a garaje, del galicismo garage, y actualmente pasa a parque, parqueadero, aparcamiento, del angloamericano parking. ¿Rechazamos la lógica evolución cultural, y renunciando al hungarismo, el galicismo y el anglo-americanismo, volvemos a usar para todo el hoy confuso término cuadra?

El que nos llegue actualmente un anglicismo de raíz latina no es nada malo, sino que enriquece nuestra expresión, de forma que de una enfermedad podemos decir que es grave, severa, seria... al igual que podemos decir que es leve, benigna, o ligera (22). 
3) Porque nos universaliza, y bendito sea el día en que todo el mundo hable la misma lengua, que si las cosas no cambian, será el inglés dentro de pocas generaciones. Sin entrar a considerar qué tienen los nacionalismos de virtud y de defecto, una cultura que quiere autoafirmarse debe hacerlo por su creatividad, y no por su impermeabilidad.

El tragar camellos, pero filtrar mosquitos, es frecuente. Y mientras por un lado tragamos y aceptamos el camello de que el término colirio, que significó en griego, latín y los romances medievales una pasta medicinal para cualquier parte del cuerpo, hoy sólo se admita para medicinas líquidas para los ojos,... por otro lado filtramos y rechazamos el mosquito grave, marcando un discutible distanciamiento con nuestras culturas circundantes.

4) Porque la RAE en su difícil quehacer, no hace la lengua sino que la recoge, y no sienta jurisprudencia, sino que aconseja. Sus cambios tienen que ser continuos. Y para terminar con un ejemplo de grave, diremos que en la terminología médica actual, cuando se hace referencia al tiempo de evolución de una enfermedad, la aguda es la corta, contrapuesta a la crónica o larga. Pero si nos atenemos estrictamente a lo que dice el último diccionario de la RAE, la enfermedad aguda es «la grave y de no larga duración», con lo que para un riguroso y grave lingüista, un orzuelo pasajero dejaría de poderse denominar meibomitis aguda por ser generalmente de escasa severidad.

\section{BIBLIOGRAFÍA}

1. Abreviaturas de autores latinos citados: Cæs. (Cayo Julio César siglo I aC). Cic. (Marco Tulio Cicerón. Siglo I aC). Hor. (Quinto Horacio Flaco, siglo I aC). Liv. (Tito Livio, siglos I aC y dC). Lucr. (Tito Lucrecio Caro, siglo I aC). Mart. (Marco Valerio Marcial, siglo I dC). Nep. (Cornelio Nepote, siglo I dC). Ov. (Publio Ovidio Nasón, siglos I $a C$ y dC). Plin. (Cayo Plinio, siglo I dC). Plaut. (Tito Maucio Plauto, siglos III y II aC). Quint. (Marco Fabio Quintiliano, siglos I y II dC). Suet. (Cayo Suetonio Tranquilo, siglos $I$ y $I I d C$ ). Ter. (Publio Terencio Afer, siglo II $a C$ ).
2. Blánquez, Fraile, A. Diccionario latino-español. Barcelona: Ed. Sopena; 1985: 706-707, 1432-1433 y 1438.

3. Palencia A. Universal vocabulario en latín y en Romance collegido por el cronista Alfonso de Palentia. Sevilla, 1490. Facsímil de la Asociación de Academias de la Lengua Española. Madrid. 1967.

4. Nebrija EA. Vocabulario español-latino. Salamanca 1495. Facsímil de la RAE de 1951.

5. Real Academia Española. Diccionario de Autoridades. Madrid: Impr. de la RAE. Tomo IV, 1734, y Tomo VI, 1739.

6. Real Academia Española. Diccionario de la lengua castellana. Madrid: Impr. Ibarra. 1780. Facsímil de la RAE. 1991.

7. Terreros y Pando E. Diccionario castellano con las voces de ciencias y artes. Madrid: Impr. Vda. de Ibarra. Tomo II, 1787: 236-237; Tomo III, 1788: 483.

8. Real Academia Española. Diccionario de la lengua española. Madrid: Impr. de la RAE. $21^{a}$ edición. 1992.

9. Real Academia Española. Diccionario de la lengua española. Madrid: Impr. de la RAE. 22 a edición. 2001.

10. Furnari S. Tratado práctico de las enfermedades de los ojos. Barcelona: Impr. La Prosperidad. 1847.

11. del Toro y Quertiellers C. Tratado de las enfermedades del ojo y de sus accesorios. Cádiz: Tip. La Mercamntil. 1879.

12. Charlín Correa C. Tratado de clínica oftalmológica. Barcelona: Salvat. 1925.

13. Ramón y Cajal S, Tello y Muñoz JF. Manual de anatomía patológica. Madrid: Tipog. Artística. 1930.

14. Meyer E. Tratado práctico de enfermedades de los ojos. Traducción de P.M. Brun. Madrid: Ed. Moya. 1875.

15. Argañaraz R. Manual práctico de oftalmología. Buenos Aires: Ed El Ateneo. 1948.

16. Arruga Liró H. Cirugía ocular. Barcelona: Ed. Salvat. 1950.

17. The concise Oxford dictionay of current English. Oxford: Oxford University Press. $4^{a}$ edición. 1951:528 y 1144.

18. Murube J. Influence of the angloamerican language in the language of Spanish speaking ophthalmologists. Madrid: Ed. Tecnimedia. 1998.

19. Corominas J, Pascual JA. Diccionario crítico etimológico castellano e hispánico. Madrid: Ed. Gredos. Tomo V. 1983; 235.

20. Real Academia Española-Asociación de Academias de la Lengua Española. Diccionario Panhispánico de dudas. Madrid: Santillana Ediciones Generales. 2005; 599.

21. Seco MA, Andrés O, Ramos G. Diccionario del español actual. Madrid: Grupo Santillana Edic. Vol.II; 2005; 4101.

22. Corripio F. Gran diccionario de sinónimos. Barcelona: Ed B.S.A. 1990. 